\title{
Terrain Modelling for Planetary Exploration
}

\author{
Ioannis Rekleitis, Jean-Luc Bedwani, Sébastien Gemme, Tom Lamarche, and Erick Dupuis \\ Canadian Space Agency, Space Technologies \\ 6767 route de l'aéroport, Saint-Hubert (Québec), J3Y 8Y9, Canada \\ FirstName.LastName@space.gc.ca
}

\begin{abstract}
The success of NASA's Mars Exploration Rovers has demonstrated the important benefits that mobility adds to planetary exploration. Very soon, mission requirements will impose that planetary exploration rovers drive autonomously in unknown terrain. This will require an evolution of the methods and technologies currently used. This paper presents our approach to $3 D$ terrain reconstruction from large sparse range data sets, and the data reduction achieved through decimation. The outdoor experimental results demonstrate the effectiveness of the reconstructed terrain model for different types of terrain. We also present a first attempt to classify the terrain based on the scans properties.
\end{abstract}

\section{Introduction}

The recent success of the Mars Exploration Rovers "Spirit" and "Opportunity" has demonstrated the important benefits that mobility adds to landed planetary exploration missions. The recent announcement by NASA to increase its activities in planetary exploration (via Moon and Mars missions) and the ESA Aurora program will certainly result in an increase in the number of robotic vehicles roaming on the surface of other planets. In particular, robotics will play a critical role in the NASA's Mars Science Laboratory [4] and ESA's Exo-Mars [9, 8]. The current stateof-the-art in control of planetary rovers requires intensive human involvement throughout the planning portion of the operations. Unless the terrain is relatively easy to navigate, rovers are typically limited to traverses on the order of a few tens of meters. Recently, the Mars Exploration Rovers "Spirit" and "Opportunity" have managed to conduct traverses on the order of 100 meters per day.

The next rover missions to Mars are the "Mars Science Laboratory" (MSL) [10] and ESA's ExoMars [8]. Both of these missions have set target traverse distances on the order of one kilometre per day. Both the MSL and ExoMars

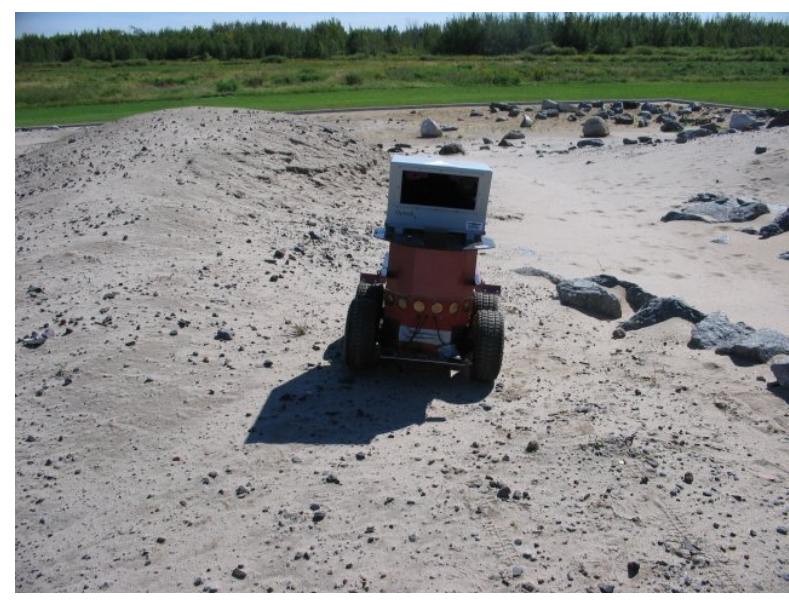

Figure 1. The Mars terrain with our modified P2AT rover.

rovers are therefore expected to drive regularly a significant distance beyond the horizon of their environment sensors. Earth-based operators will therefore not know a-priori the detailed geometry of the environment and will thus not be able to select via points for the rovers throughout their traverses. Path and trajectory planning will have to be conducted on-board, which is an evolution from the autonomy model of the MERs.

One of the key technologies that will be required is the ability to sense and model the 3D environment in which the rover has to navigate. The current sensing model is based on passive vision. We are currently developing the capabilities of active vision systems such as 3D laser range finders. The typical operational scenario used for our experimentation is based on the following assumptions. The rover, see Fig. 1, has rough a-priori knowledge of its surroundings in the form of a low-resolution terrain map. The rover iteratively takes local scans of its surroundings, localizes, plans a path, and navigates segments of the planned path. The local scans are used for three purposes: planning a local path to the next way-point while avoiding known obstacles, re- 


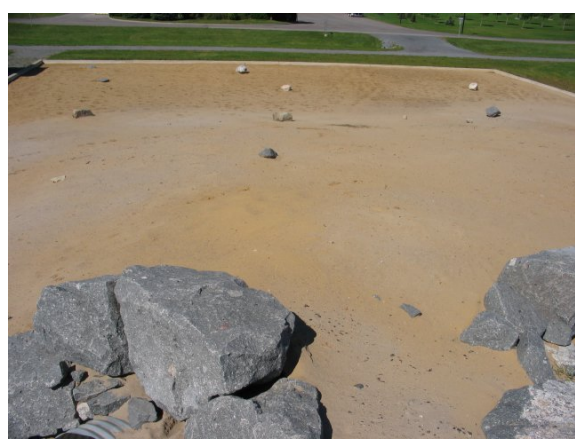

(a)

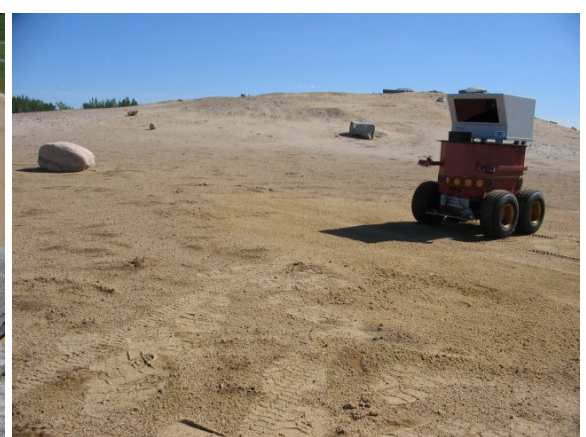

(b)

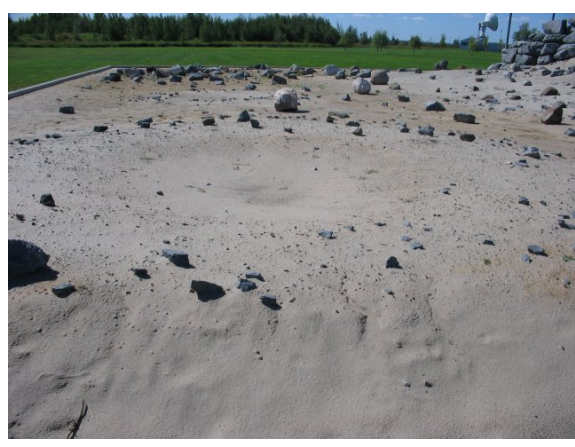

(c)

Figure 2. Different views of the Mars terrain where the scans were taken. (a) Flat area with few obstacles, seen from the top of the hill; (b) gentle slope up the hill from the flat area; (c) flat area with many obstacles.

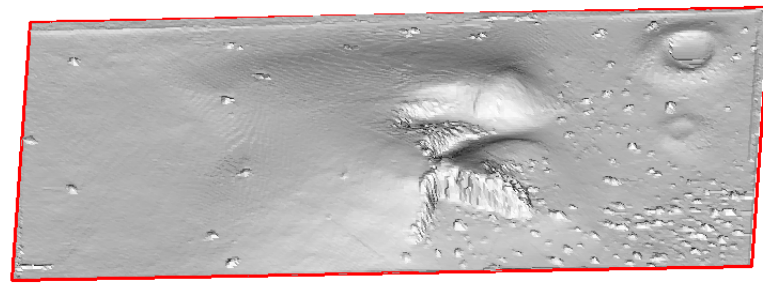

Figure 3. A complete model of the Mars terrain. Figure $2 a$ is taken from the center of the terrain looking to the left; fig. $2 b$ is taken from the left side; and Fig. $2 \mathrm{c}$ is taken from the top right side, just above the crater.

fining the localization knowledge throughout the trajectory by observing again the traversed terrain, and finally constructing an atlas $[2,7]$ of detailed terrain maps to be added to the coarse terrain model. Central in all three uses is the processing and analysis of the scan-based terrain model.

This paper presents the terrain modeling approach taken at CSA for planetary exploration. In particular, we present an analysis of the properties exhibited by a variety of terrain scans taken in our Mars emulation terrain (Mars terrain). The terrain is modeled as an irregular triangular mesh. Figure 2 presents views of the different types of Martian terrain modelled at CSA. On one side, there is a flat area with few obstacles that is easy to navigate through but hard to localize; see Fig. 2a. A small hill that has a gentle slope on one side, see Fig. 2b, and a cliff on the other side, see Fig. 1, occupies the center of the terrain. Finally a flat area with many obstacles and two small craters are at the other side of the hill; see Fig. 2c. Figure 3 shows a model of the whole Mars terrain.
The next Section presents the related work. The terrain modelling algorithms and statistical results on the data compression achieved by scan decimation are in Section 3. Section 4 provides an overview of different statistical properties of the terrain models and qualitative experimental results. A classification of the different terrain kinds modeled in CSA's Mars terrain based on the statistical properties presented in the Section 4 are provided in Section 5. Finally, the last Section contains concluding remarks and future work.

\section{Background}

Currently, the most advanced exploration robots for planetary exploration are the Mars Exploration Rovers (MER) "Spirit" and "Opportunity". These rovers have successfully demonstrated, on Mars, concepts such as visual odometry and autonomous path selection from a terrain model acquired from sensor data. The main sensor suite used for terrain assessment for the MER has been passive stereo vision [11]. The models obtained through stereo imagery are used both for automatic terrain assessment and for visual odometry.

In the case of automatic terrain assessment, the cloud of $3 \mathrm{D}$ points is used to evaluate the traversability of the terrain immediately in front of the rover, defined as a regular grid of square patches. In the case of visual odometry, the model is used to identify and track features of the terrain to mitigate the effect of slip. [5]

Our work focuses on a different sensing modality, a laser range finder which return accurate geometric information in three dimensions. As such we do not have to address the problems arising from harsh lighting conditions. In addition, the sensor already provides three-dimensional data in the form of a 3D point cloud. We are taking advantage of 


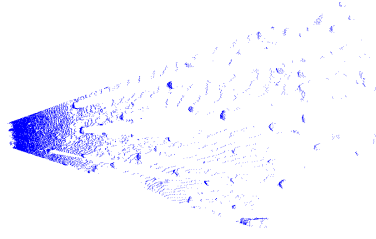

(a)

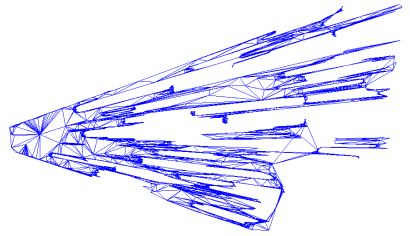

(b)
Figure 4. (a) A LIDAR scan; (b) the same scan decimated and represented as a triangular mesh.

this sensor characteristic to build a terrain model that preserves the geometry of the terrain but that is also readily usable for path planning and navigation.

\section{Terrain Modelling}

Central to planetary exploration is the sensing and modeling of the terrain through which the rover will navigate. The terrain sensor used on CSA's experimental test-bed is a commercial LIght Detection And Ranging (LIDAR) sensor: an ILRIS-3D sensor from Optech. The main advantage of the LIDAR is that it directly provides a $2.5 \mathrm{D}$ point cloud giving the $x-y-z$ coordinates of the terrain in its field of view. The sensor has a range of over 1 kilometre but the data is trimmed down to approximately 50 metres. Some of the specific challenges that must be addressed by the terrain modelling software are the high volume of data (each LIDAR scan can contain as many as $500 \mathrm{~K} 3 \mathrm{D}$ points at full range), and the highly non-uniform density of the scans due to the fact that the sensor is mounted at a grazing angle. Indeed, to increase the challenges associated with over-thehorizon navigation, the sensor is mounted directly on top of the rover, approximately $50 \mathrm{~cm}$ off the ground; see Fig. 1. This has the effect of shortening the horizon and introducing severe occlusions in the presence of obstacles; see Fig. 4a.

Since a point cloud is not an appropriate structure for path-planning and navigation, a different representation was chosen. One of the requirements of the selected representation is that it must be compatible with navigation algorithms and that it must preserve the scientific data contained in the terrain topography. In addition, the resulting model must be compact in terms of memory usage since the model must reside on-board the rover.

To fulfil these requirements, the irregular triangularmesh terrain representation was chosen. One of the main advantages of the irregular triangular-mesh over the classical digital elevation maps (DEM) is that it inherently supports variable resolution. It is therefore possible to decimate the data set, modelling details of uneven areas with

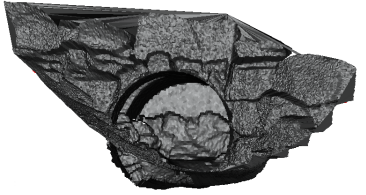

(a)

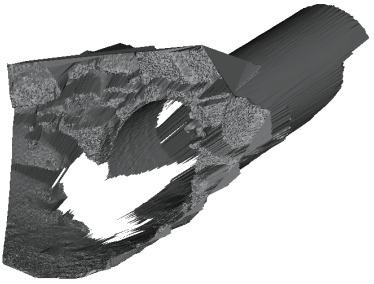

(b)
Figure 5. (a) The model of the entrance of a cave/tunnel in the Mars terrain, single scan; (b) The same scan viewed from above shows the model of the pipe that forms the inside of the tunnel.

high precision, while simplifying flat areas to just a few triangles; see Fig. 4b for a decimated triangular mesh of the data points presented in Fig. 4a. The decimated mesh drastically reduces the overall memory requirements for a given terrain. The irregular triangular-mesh (ITM) representation also has advantages compared to other variable resolution representations such as quad-trees or other traversability maps, which remove all science content from the topographical data. In addition, both DEM and quad-trees are $2.5 \mathrm{D}$ representations. Therefore, they do not support concave geological structures like overhangs and caverns, which pose no problem to the irregular triangular mesh. Oct-trees provide 3D representation but at finite resolution, as such the model appears "pixelized" even at the highest resolution, moreover, updates could be really costly computationally. Figure 5 show two views of the data collected at the entrance of an artificial cave/tunnel at our Mars terrain. It is worth noting that, as can be seen at Figure 5b, the inside of the tunnel -made of a large drain pipe- was modelled as a cylinder by many small triangles. Such details were possible to model due to our representation choice. The implementation of the terrain modelling using a triangular mesh is done using the Visualisation Toolkit [1]. The Delaunay triangulation algorithm is used on the point cloud and then triangles, termed shadow triangles, that appear behind obstacles, thus not visible from the sensors POV, are removed.

As mentioned earlier, an important step is the decimation of the dense point cloud. The decimation algorithm was run repeatedly over the rich data sets obtained in the CSA's Mars-like terrain. The scans were taken using the ILRIS LIDAR in the same configuration that is used for the navigation experiments. The terrain is representative of what a rover would encounter in several locations on the surface of Mars. There are two parameters guiding the decimation, one is the maximum allowed error and the other one is the 


\begin{tabular}{|l|r|r|r|r|r|}
\hline \multicolumn{2}{|c|}{} & \multicolumn{4}{|c|}{ Target Decim. Ratio } \\
\hline & Orig. Scans & $75 \%$ & $80 \%$ & $90 \%$ & $95 \%$ \\
\hline Avg \# of Points & 31233.6 & 8072.3 & 6527.9 & 3435.8 & 2083.7 \\
\hline Avg \# of Triangles & 61646.0 & 15410.6 & 12328.3 & 6191.5 & 3584.1 \\
\hline Avg Effective Decim. Ratio & N/A & $74.06 \%$ & $79.00 \%$ & $88.86 \%$ & $93.01 \%$ \\
\hline STD Effective Decim. Ratio & N/A & $0.70 \%$ & $0.74 \%$ & $1.15 \%$ & $2.30 \%$ \\
\hline
\end{tabular}

Table 1. Properties of Decimated Terrain Scans, Acceptable Error $1.5 \mathrm{~cm}$

decimation target ratio. Different decimation target ratios were used with a set maximum absolute error of $1.5 \mathrm{~cm}$. Table 1 presents the cumulative results for 91 scans. Target decimation ratios of $75 \%, 80 \%, 90 \%$, and $95 \%$ were used, the average achieved ratio for the maximum allowed error is presented together with the average reduction in the number of points and the number of triangles for every case.

Another measure for the appropriateness of decimation is the distribution of the surface slope. In particular we are interested in preserving the data points and triangles of areas with high curvature while eliminating points that are co-planar. The angle of the surface normal to the z-axis is selected as a measure for each triangular patch. Figure 6 presents the slope distribution as a function of the number of triangles for different decimation ratios together with the distribution of slopes for the original scan. The bar provides the distribution of the original scan and it is the same in all four sub-figures. The *-line presents the distribution for different decimation ratios. As can be seen the percentage of low slope triangles decreases as the rate of decimation increases, thus the number of flat triangles decreases while the higher slope triangles stay the same thus increasing in percentage.

\section{Statistical Analysis of Terrain Models}

Central to the autonomous planetary operations is the ability to analyze the data collected. In particular, for terrain models the rover should be able to analyze if the scan obtained is first, adequate for navigation i.e. does the scan cover enough area to navigate through? and second, to be able to classify the type of terrain. The experimental terrain used at CSA has an area of sixty by thirty meters, as the scans were taken in a variety of positions around the Mars terrain with a range of fifty meters, large parts of the scans included areas outside the Mars terrain. In several cases, man made structures such a small buildings, snow-poles, cars, and a wind generator were also scanned. In order to characterize only Mars-like terrain, the scans were cropped roughly to the area of the Mars terrain.

The effective range of the scan, also called depth of field, is the first measure as it determines how far the sensor was able to "see". For robustness the farthest hundred points are removed, and then the longest distance from the sensor to the data points defines the effective range. As such when the rover is positioned right in front of a steep hill or a big obstacle the effective range is very short, while when the rover views a flat area it can sense to the limits of the sensor.

The total area covered by the scan is one measure of the effectiveness of the scan. The percentage of the effective area covered by the scan to the total viewable area is another measure of the quality of the scan and also of the terrain type. The percentage of the area covered is measured in two ways, using the effective range of the scan and also using the maximum range of the sensor, currently at fifty meters. Therefore, the percentage of the area covered measures obstructions in the field of view, as the areas behind obstacles appear empty in the terrain model.

A similar measure for coverage is the perimeter of the scan, which is the total length of the triangle edges that are bordering un-modelled/empty areas. As such the perimeter consists of the boundary to the outside of the scan, and the boundary to the "holes"/"shadows" inside the scan.

The morphology of the terrain can be described by many parameters, we are using the maximum elevation to establish how hilly the terrain is, either, in absolute terms or in conjunction with the effective range. Furthermore, the distribution of the slope for all the triangles of the mesh provides another set of parameters for classifying the terrain type. In particular, the average slope, measured as the angle of the normal of the triangle to the z-axis, together with the standard deviation of the slopes provides a good estimate on the terrain type. If the average slope is small the terrain is flat, and the standard deviation is an indication of the existence of obstacles in the field of view. High standard deviation means that many obstacles with a variety of slopes exist. Finally, if the average slope is higher then this is an indication of a scan that covers the hill. The elevation of the scan is an absolute measure of the existence of the hill in the scan but it is not robust as even a small portion of the hill if it is viewed would contribute to a high elevation. Table 2 presents the parameters for some representative scans of the three types of terrains modelled. As can be seen, the variation in the parameter values is very high. 


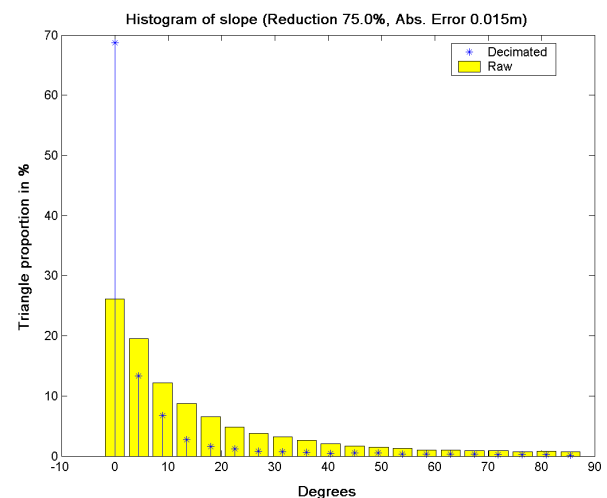

(a)

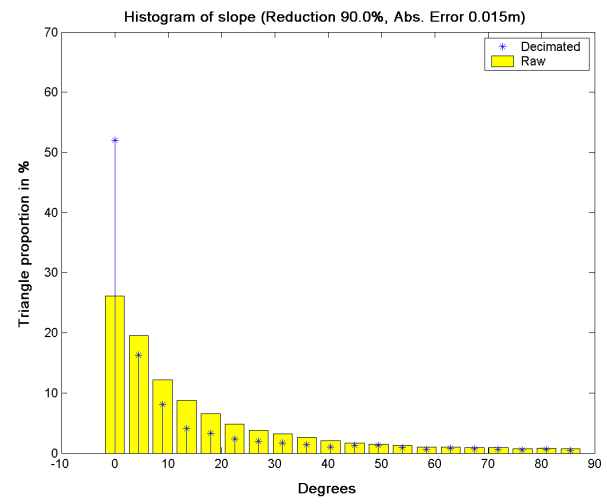

(c)

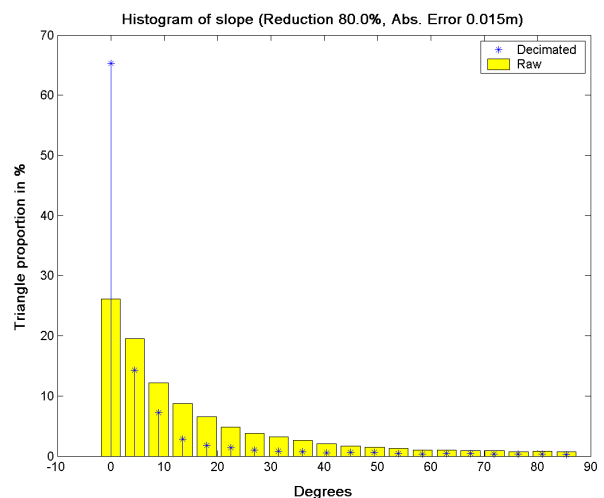

(b)

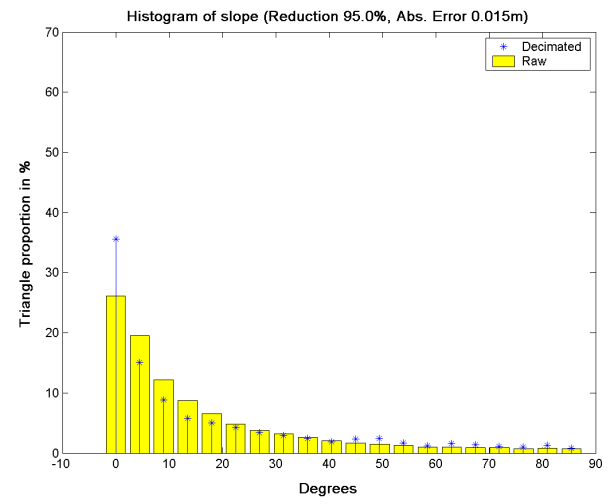

(d)

Figure 6. Histogram of the slope distribution as a function of the number of triangles for different decimation ratios. The bar provides the distribution of the original scan and it is the same in all four sub-figures. The *-line presents the distribution for different decimation ratios. (a) $75 \%$, (b) $80 \%$, (c) $90 \%$, and (d) $95 \%$ decimation ratio.

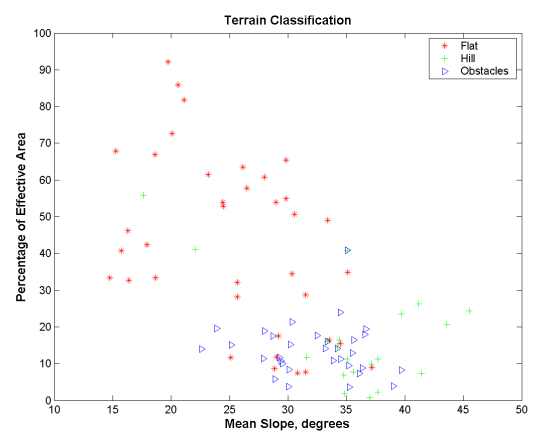

(a)

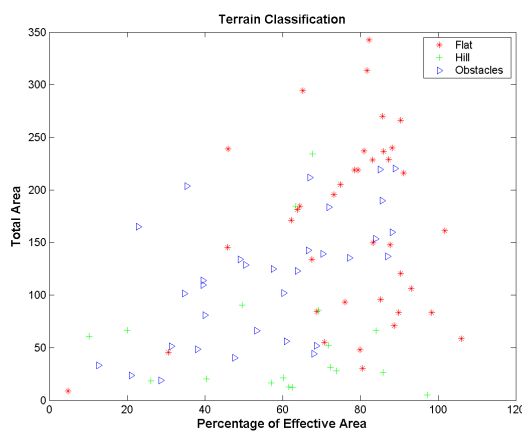

(b)

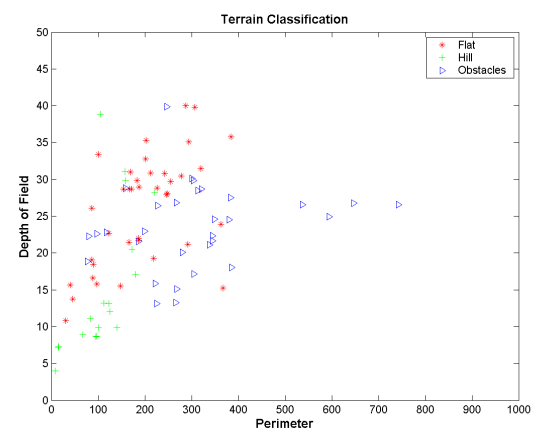

(c)

Figure 7. Terrain Classification using different parameters. (a) The effective area covered vs the mean slope. (b) The effective area covered vs the total area covered. (c) The depth of field vs the perimeter. 


\begin{tabular}{|l|r|r|r|}
\hline & Flat & Hill & Obstacles \\
\hline Perimeter & $171.3 \mathrm{~m}$ & $94.7 \mathrm{~m}$ & $742.9 \mathrm{~m}$ \\
\hline Elevation & $0.9 \mathrm{~m}$ & $2.2 \mathrm{~m}$ & $3.5 \mathrm{~m}$ \\
\hline Depth of field & $28.6 \mathrm{~m}$ & $8.6 \mathrm{~m}$ & $26.5 \mathrm{~m}$ \\
\hline Total area & $228.6 \mathrm{~m}^{2}$ & $26.6 \mathrm{~m}^{2}$ & $101.4 \mathrm{~m}^{2}$ \\
\hline Effective Area & $83.1 \%$ & $85.8 \%$ & $34.7 \%$ \\
\hline Mean slope & $22.3^{\circ}$ & $38.9^{\circ}$ & $24.6^{\circ}$ \\
\hline Slope Variance & $8.6^{\circ}$ & $8.5^{\circ}$ & $10.8^{\circ}$ \\
\hline
\end{tabular}

Table 2. Parameters for representative scans of the three different types.

\section{Terrain Classification}

The Mars terrain at CSA can be roughly divided in three different types. A flat area with few obstacles at one side of the terrain; see Fig. 2a for a picture and Fig. 3 for the model. A flat area with two small craters and many obstacles (rocks) at the other side of the terrain; see Fig. 2c and Fig. 3 accordingly. Finally the center of the terrain has two small hills with a gentle slope to one side and a small cliff to the other; see again Fig. 2b and Fig. 3. The ninety one scans have been classified manually as belonging to one of the three terrain types. Unfortunately there are two problems with this classification, first, there are many scans that cover parts of different terrain types, therefore, features overlap, such as long effective range with high elevation when a scan is taken facing up a gentle slope up to the top of the hill; and second, due to localization error some man-made constructs near the borders of the Mars terrain remain in the cropped scans. The above mentioned issues appear as outliers in our classification of the terrain type from the properties of the scan.

Figure 7 presents different classification parameters for the complete set of scans. The mostly flat scans are presented as (red) stars, the scans of the hill as (green) crosses, and the scans with many obstacles as (blue) triangles. Figure $7 \mathrm{a}$ presents the mean slope versus the percentage of area covered, flat terrain occupies the top left part of the graph, while the hilly terrain occupies the lower right, the terrain with obstacles is in the middle with outliers mixing with the flat and the hilly terrain scans. Figure $7 \mathrm{~b}$ presents the area measures (total and percentage), the division here is more along the vertical as the scans of the hill cover less total area, and the flat terrain typically has large total and in percentage area coverage. The existence of obstacles tends to reduce the area covered in general. Finally, Fig. $7 \mathrm{c}$, presents length parameters such as depth of field and perimeter. The hilly terrain is at the lower left corner, with short depth of field and perimeter; the presence of obstacles usually increases the length of the perimeter, sometimes to really high values; unobstructed flat terrain has usually long

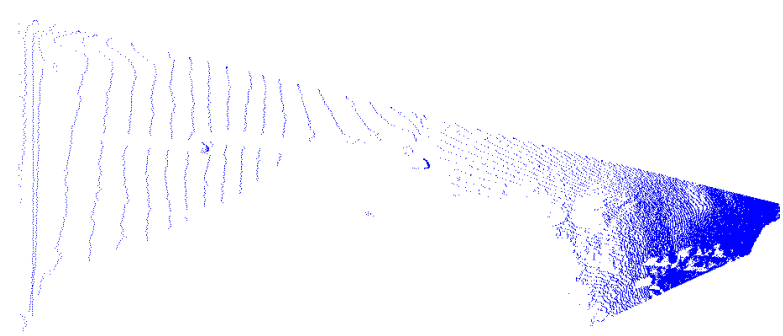

Figure 8. A scan covering flat terrain with two obstacle, the side of the hill, and large occlusions.

\begin{tabular}{|l|r|}
\hline Perimeter & $247.0 \mathrm{~m}$ \\
\hline Elevation & $2.6 \mathrm{~m}$ \\
\hline Depth of field & $39.8 \mathrm{~m}$ \\
\hline Total area & $203.7 \mathrm{~m}^{2}$ \\
\hline Effective Area & $35.4 \%$ \\
\hline Mean slope & $25.1^{\circ}$ \\
\hline Slope Variance & $8^{\circ}$ \\
\hline
\end{tabular}

Table 3. The parameters for the scan presented in Fig. 8.

depth of field.

As can be easily seen from the above discussion a clear classification is not easy, as several scans cover areas that belong to two different types. For example, the scan presented in Fig. 8 contains a view of the flat terrain with two obstacles at the left side, but on the right side a high slope that occludes a large part of the field of view is prominent. Table 3 presents the different parameters for the scan seen in Fig. 8, a long field of view is combined with low percentage of area covered and also with a high elevation.

\section{Conclusions}

In this paper we presented our approach to terrain modelling for planetary exploration. Terrain modeling is a fundamental issue, we propose the irregular triangular mesh as a compact representation that maintains the topography of the terrain. The size reduction via decimation, together with a variety of properties of the different scans was presented. We have run the decimation and parameter estimation algorithms on all 91 scans collected in CSA's Mars emulation terrain. The decimation provide volume reduction up to 93\% for terrains representative to the Mars topography for bounded error. The choice of the irregular triangular mesh was able to model true 3D structures which are more complex than 2.5D. Furthermore, the statistical analysis seems 


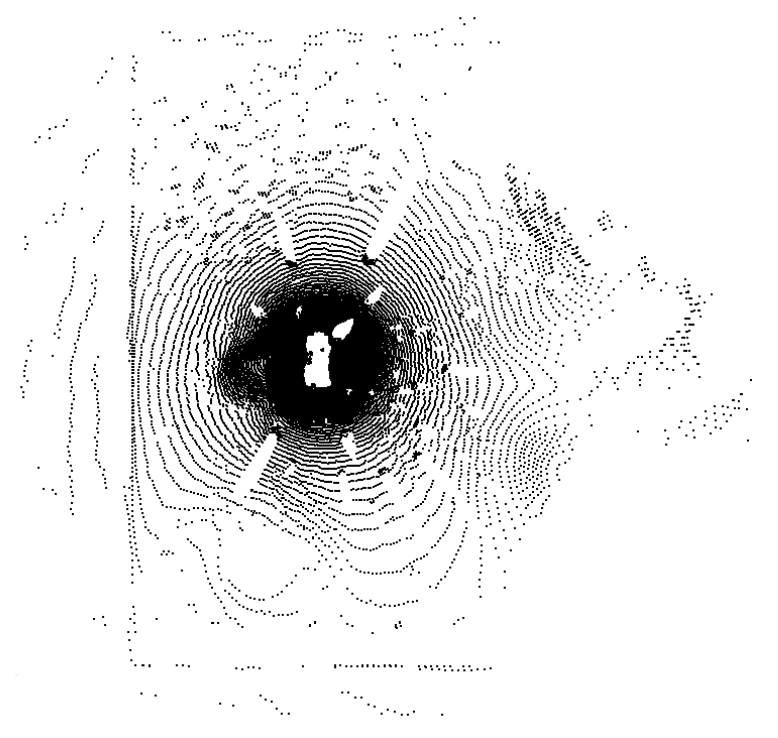

Figure 9. Laser Scan using a SICK laser mounted on a pan-unit.

to enable terrain classification.

We are currently developing a 3D scanning laser system using a SICK laser mounted on a pan-unit. The resolution and range of this new sensor is reduced compared to the LIDAR sensor from Optech, but it is much lighter and it has a 360 degrees field of view. The larger FOV is a necessary improvement as the current narrow FOV appeared to be very restrictive for navigation when there are obstacles near the sensor. The new sensor is also mounted at a low grazing angle, as evidenced by the long shadows (lack of data) in the scan; see Fig. 9. The low mounted sensor is a strong constraint as several times the horizon was very close, thus requiring frequent scans. During the next testing period we are going to continue the planetary exploration work using this new sensor. Furthermore, we are working on automated data interpretation as in several cases the presence of obstacles created narrow corridors that were barely navigatable by the rover.

The advantages of active vision for space applications as demonstrated by the successful use of Neptecs Laser Camera System (LCS) sensor on mission (STS-105) and in every other space shuttle mission since then, are numerous. The XSS-11 mission also used an active vision system from Optech $[3,6]$ for long range rendezvous. In planetary exploration in particular, such a sensor provides accurate data independent of the lighting conditions and allows for accurate mapping of a variety of geological formations. We are confident that the technologies presented in this paper are going to contribute greatly towards the success of future missions.

\section{References}

[1] Kitware inc. visualization toolkits. http://www.vtk.org, Website (accessed: September 2005)., 2005.

[2] M. Bosse, P. Newman, J. Leonard, and S. Teller. Simultaneous localization and map building in large-scale cyclic environments using the atlas framework. International Journal of Robotics Research, 23(12):1113-1139, December 2004.

[3] E. Grossman and K. Costa. Small, experimental satellite may offer more than meets the eye. Inside The Pentagon, December 42003.

[4] S. Hayati, G. Udomkesmalee, and R. Caffrey. Initiating the 2002 mars science laboratory (msl) focused technology program. In IEEE Aerospace Conference, Golden, CO, USA, March 2004.

[5] A. M. Howard and E. W. Tunstel, editors. Intelligence for Space Robotics, chapter MER Surface Navigation and Mobility. TSI Press, 2006.

[6] J. Lewis. Space weapons in the 2005 US defense budget request. In Workshop on Outer Space and Security, Geneva, Switzerland, 2004.

[7] B. Lisien, D. Morales, D. Silver, G. Kantor, I. M. Rekleitis, and $\mathrm{H}$. Choset. The hierarchical atlas. IEEE Transactions on Robotics, 21(3):473-481, June 2005.

[8] J. Vago. Overview of exomars mission preparation. In 8th ESA Workshop on Advanced Space Technologies for Robotics and Automation, Noordwijk, The Netherlands, November 2004.

[9] M. Van Winnendael, B. Gardini, R. Roumeas, and J. Vago. The exomars mission of esa's aurora programme. In Earth and Space 2004 - Ninth Biennial Conference of the Aerospace Division, Houston, Texas, USA, March 2004.

[10] R. Volpe. Rover functional autonomy development for the mars mobile science laboratory. In IEEE Aerospace Conference, Big Sky, MT, USA, 2006.

[11] J. Wright, A. Trebi-Ollennu, F. Hartman, B. Cooper, S. Maxwell, J. Yen, and J. Morrison. Terrain modelling for in-situ activity planning and rehearsal for the mars exploration rovers. In IEEE International Conference on Systems, Man and Cybernetics, volume 2, pages 1372 - 1377, 10-12 Oct. 2005. 